\title{
The Role of Sentinel Lymph Node Biopsy in the Management of Breast Cancer
}

\author{
MM SAHA $^{\mathrm{a}}$, MM HOSSAIN ${ }^{\mathrm{b}}, \mathrm{S}^{\mathrm{S}} \mathrm{SHA}^{\mathrm{c}}$
}

\begin{abstract}
Summary:
The sentinel lymph node (SLN) is defined as the first node(s) receiving lymphatic drainage from a primary tumour. A promising alternative to axillary lymph node dissection (ALND) is sentinel lymph node biopsy. SLN biopsy has been introduced as a technique to identify axillary lymph node most likely to contain tumour cells metastasizing from a primary carcinoma of breast. Several methods of identifying the SLN exists, including the use of radioactive tracer, lymphazurin dye or combination of the two via intraparenchymal and/or intradermal, peritumoral or periaerolar injection sites. Intraoperative evaluation of SLNS are done by performing FS(Frozen Section) on all the lymph nodes after serially sectioning them at 3-4mm intervals; at least 2 levels are cut of all the sentinel lymph nodes. In addition, touch preparation cytology(TP) smear may also be made for evaluation. The limitations of SLNB is that a proportion of patients who have metastasis limited to the $S L N$ can be predicted when there is a combination of tumour
\end{abstract}

Introduction:

Breast cancer is the leading cause of death in women with over 3,00,000 deaths annually world wide ${ }^{1}$. Axillary nodes status is the best single predictor of disease outcome in patient with early stage breast cancer. It has been shown that the presence of axillary node metastasis reduces the patient's survival by 28$40 \%{ }^{2}$ and the likelihood of treatment failure increases with the increase number of positive axillary nodes ${ }^{3}$. Furthermore the removal of axillary nodes also improves local and regional control, which leads to better over all survival. Axillary lymph node dissection (ALND) has been the only reliable method for

a. Dr. Moni Mohan Saha, Professor \& Director, Shaheed Sk Abu Naser Specialized Hospital, Khulna

b. Dr. Md. Mokter Hossain, Assistant Professor, Department of Pathology, Khulna Medical College, Khulna

c. Dr. Sukumar Saha, Junior Consultant, Shaheed Sk Abu Naser Specialized Hospital, Khulna.

Address of Correspondence: Dr. Moni Mohan Saha, Professor \& Director, Shaheed Sk Abu Naser Specialized Hospital, Khulna.

Received: 18 June, 2013

Accepted: 16 July, 2014 size $<1.0 \mathrm{~cm}$, the absence of lymphovascular invasion and micrometastatic disease $(<0.2 \mathrm{~cm})$ in $S L N$. However for patients with large breast cancer, the role of SLNB is controversial. Early studies of SLNB in large breast cancer patients demonstrated a high (8-18\%) false negative rate, with the accuracy worsening with the increasing size. Excision of SLNs have an extremely low morbidity and a high degree of staging accuracy. A tumour-free SLN virtually excludes lymphatic involvement of the entire regional lymphatic basin. More than 50 observational studies of SLNB validated by a back up ALND demonstrate that SLNB is feasible, accurate and suitable for virtually all patients with operable clinically node negative disease. Sentinel lymph node biopsy not only provide prognostic information, but also aims to guide adjuvant therapy without the untoward side effects of complete axillary dissection.

Key words: Sentinal lymph node, Management, Breast Cancer.

(J Banagladesh Coll Phys Surg 2014; 32: 211-217)

determining lymph node status ${ }^{4}$. However the clinical importance of axillary control and the extent of axillary surgery remain controversial issues. Previous studies on sentinel lymph node biopsy (SLNB) validated by a back up axillary lymph node dissection (ALND) confirm that SLND is both feasible and accurate, reliably detecting axillary metastasis in $97 \%$ of all patients and $93 \%$ of node positive cases ${ }^{5}$.

The surgical management of invasive breast cancer has included complete removal of the tumour, with documentation of negative margins by either mastectomy or breast conserving surgery and synchronous complete axillary lymph node dissection (ALND). However the complication rate of ALND are reported to be $20-55 \%$. This complications include lymphedema, sensory nerve damage, haemorrage and post operative seroma formation as well as need for general anaesthesia ${ }^{6}$. In invasive breast carcinoma, axillary lymph node metastasis is seen in only $40 \%$ patients undergoing $\mathrm{ALND}^{7}$, and is even less in early breast cancer; therefore ALND is not needed in the 
majority of the patients. Since ALND is also associated with significant morbidity. A promising alternative to ALND is sentinel node (SLN) biopsy for axillary staging of invasive breast cancer. Sentinel lymph node mapping is a minimally invasive procedure and is very effective and accurate method of evaluating the regional lymph nodes in breast cancer patients. It is noteworthy that a pathologically negative sentinel lymph node predicted the absence of metastasis. The reminder of regional lymph node basin with about a $98 \%$ degree of certainty ${ }^{8}$. The sentinel lymph node is defined as the first node(S) receiving lymphatic drainage from a primary tumour. It is therefore the node most likely to contain metastatic breast carcinoma. A tumour-free SLN virtually excludes lymphatic involvement of the entire regional lymphatic basin. Sentinel lymph node biopsy (SLNB) not only provides prognostic information but also aims to guide adjuvant therapy without the untoward side effects of complete axillary dissection. More than 50 observational studies of SLNB validated by a back up ALND demonstrate that SLNB is feasible, accurate and suitable for virtually all patients with operable, clinically node negative disease.

\section{Historical perspective:}

The concept of sentinel node was first described by Cabanas R in 1977 in the context of penile carcinoma9 ${ }^{9}$. In 1992 Morton and his colleagues reported a blue dye technique for lymphatic mapping and sentinel node biopsy in clinically node negative melanoma patients ${ }^{10}$ and later by Van der veen and colleagues with lympho scintigraphy to select melanoma patients for regional lymph node dissection ${ }^{11}$. In the decade since the pioneering reports of Krag et al and Giuliano et al, SLNB has become a new standard of care for axillary lymph node staging in patients with breast carcinoma ${ }^{12}$. Lymphatic mapping and SLNB are clearly changing the paradigm for the treatment of breast cancer.

\section{Sentinel lymph node biopsy technique:}

Sentinel lymph node biopsy is simple technique which uses subdermal or peritumoral injection of vital blue dye or radio-labelled colloid or both substances together to identify the first lymph node draining the primary tumour. Several methods of identifying the SLN exists, including the use of radioactive tracers, lymphazurin dye or combination of the two via intraparenchymal and /or intradermal, peritumoral or periaerolar injection sites. In 1998 O’Hea et al ${ }^{13}$ reported their initial experience at Memorial Sloan-Kettering cancer centre that the blue dye and radioisotope were the complementary technique in SLN mapping biopsy in breast cancer patients, and that the overall success of this procedure was maximized when the two are used together(93\%) as compared to when used alone(75 \& 88\% respectively) in SLND mapping in the breast cancer patients. With increasing experience of the breast surgical oncologists with this procedure over the last decade. SLNB is usually straight forward and a simple operation which has become standard of care for surgical management of breast cancer. Before the surgical resection of breast tumour(lumpectomy or mastectomy) blue dye and redioactive tracer is injected into the breast in a peritumoral, intradermal or periaerolar manner. Isotope counts are taken using a hand held-gamma probe for supraclavicular, infraclavicular, parasternal and axillary areas. More than a $95 \%$ of the time a hot spot is found in the ipsilateral axilla. An axillary incision is made the surgeon identifying all blue and /or hot nodes as well as palpable nodes. These are removed until the axillary background counts fall below a threshold value: defined as the background count; most authors report a median of two SLN per patient ${ }^{14}$. A success of $90-95 \%$ in finding the SLN and no more than 5-10\% false negative results would seem reasonable targets for validation trials ${ }^{15}$. Results of identifying SLN using different technique are shown in Table-1. Intraparenchymal injection was the first technique described and is the most widely accepted. Recent data suggest that the intradermal technique is highly accurate and may increase the SLN identification rate. SLN localization was successful in $97 \%$ of cases with intradermal radioisotope injection and in $78 \%$ of those with intraparenchymal injection, a statistically significant difference ${ }^{16}$. Knox and Ley compare the intraparenchymal Vs intradermal injection of radioisotope for identification of SLN in breast cancer patients and reported that intradermal technique may increase SLN identification and is easy to use and has acceptable false negative rate $(2 \%)^{17}$. The greater ease of SLN identification rate with intradermal injection may be due to the rich lymphatic network of skin overlying the breast as well as reduction in shine effect. Krag et al conducted multicenter validation study using radioactive tracer technique. The overall rate of identification of 
hot spots was $93 \%$ with an accuracy of $97 \%$, specificity $100 \%$ and sensitivity $89 \%{ }^{18}$.

Intra-operative evaluation of sentinel lymph nodes: Careful intraoperative palpation of the axilla is an essential component of SLN biopsy. Apart from the fact that the detection rate is approximately $80-90 \%$ in the largest series, the key problems are false negative and intraoperative examination of sentinel node. Routine frozen section (FS) examination appears to miss up to $30 \%$ of metastasis in sentinel nodes ${ }^{19}$. However the value of routine intraoperative SLN frozen section is controversial. If positive, FS has the obvious advantage of allowing an immediate axillary dissection and thereby avoiding the reoperation. On the other hand FS is costly, time consuming and subject to false negative results. Brogi et al in 2005 demonstrated that FS, touch preparation(TP) and cytological smear (CS) are equivalent for the intraoperative assessment of SLN in breast cancer (comparable sensitivities were 59\%, 57\%, $59 \%$ respectively $)^{20}$. Each method was more sensitive in detecting macrometastasis ( $>2 \mathrm{~mm}$ tumour deposite, $96 \%, 93 \%$, 93\%) than micrometastasis ( $<2 \mathrm{~mm}, 27 \%$, $27 \%$, 30\% respectively). The added benefit of combining methods is small and the failure of intraoperative assessment is largely due to an inability to detect micrometastatic disease. A study by Krogerus et $\mathrm{al}^{21}$ compared the widely used method of intraoperative evaluation of SLN which is a modification of the method introduced by Veronesi et $\mathrm{al}^{22}$ including bisecting the lymph node in its long axis and examining serial sections to their own novel method. Krogerus's method included sectioning the lymph node into thin slices (1-1.5mm thick) perpendicular to the long axis and arranged on a prefrozen tissue-Tak, frozen section from two level were then examined. With this method, they found more and smaller metastatis compared to the widely used method and also the technique was less time consuming. Recently intraoperative evaluation of SLN includes performing FS (Frozen section) on all the lymph nodes after serially sectioning them at 3$4 \mathrm{~mm}$ intervals; at least 2 levels are cut of all the sentinel lymph nodes. In addition touch preparation cytology (TP) smear may also be made for evaluation. However there exists a personal bias among pathologists in examining the cytology smears especially pathologists who are not practicising cytopathologist. TP is an additional and complementary method to FS in the intraoperative evaluation of SLN. The FS includes examining all lymph nodes bisecting them if they are more than $0.5 \mathrm{~cm}$ in thickness and cutting at least two levels of each section. The sentinel node can be examined intra-operatively by frozen section or imprint cytology, both of which have a high specificity ${ }^{23}$. Axillary node clearance can subsequently be performed if intra-operative examination of the node is positive for malignancy; thus avoiding the need for a second surgical procedure.

The introduction of SLNB has allowed focus examination of tumour biology within the sentinel node. The clinical role of immunohistochemistry (IHC) and polymerase chain reaction (PCR) in detecting micrometastatic disease remains however at an investigational stage ${ }^{24}$.

Pathologic evaluation of sentinel lymph nodes on permanent sections (Role of serial sectioning (SS) and immunohistochemistry (IHC)): while SLNB has clearly established its status and has become the new standard of care in axillary staging of breast cancer, there seems to be little consistency in the pathologic procedures used by different laboratories in their evaluation. Lymph nodes were marked as sentinel or non-sentinel. A preliminary frozen section was performed on the sentinel and non-SLNs at the Mayo clinic. If the SLN was negative for tumour, the lymph node was formalin fixed and paraffin embedded and at least four additional levels were examined. Two levels were stained with standard haematoxyline and eosin (H\&E) and an additional level stained with cytokeratin antibody, together with negative control. The SLNs removed at the university of Pennsylvania were formalin fixed, paraffin embedded and evaluated with H\&E and immunohistochemistry. All non-SLNs were evaluated with standard H\&E sections. In all cases, the size of the SLN was measured using an occulometer. A micrometastasis was defined as a tumour deposit of $<2.0 \mathrm{~mm}$. A metastasis $>2.0 \mathrm{~mm}$ was considered to be a macrometastasis. If multiple tumour deposits were present in the SLN, the sum of the tumour deposits was used to classify the metastasis as micro -or macrometastasis. In cases in which there was more than one SLN positive for tumour, the patient was grouped according to the largest size of metastasis. Primary tumour or re-excision specimens were evaluated by routine histology. Various studies have shown that 
SLNB with examination of multiple serial sections(SS) and immunohistochemistry(IHC) greatly improves the sensitivity for identifying nodal metastatis especially micrometastasis $(<0.2 \mathrm{~cm})$. It has been suggested that these more comprehensive methods of pathologic examination will find axillary lymph node metastasis in $7-42 \%$ of patients with breast carcinoma initially diagnosed as lymph node negative and that missed metastasis may be prognostically significant ${ }^{25}$. Vialo et al recommended sectioning the SLN entirely at close intervals ${ }^{26}$.

As shown in Table-2, increasing the sectioning interval from 50 micrometer to 290 micrometer may miss as many as $17.7 \%$ of micrometastatic SLN.

A study by Intra et al showed that examination of SLN by serial sectioning and IHC increases the detection rate of metastasis from, 9-33\% in infiltrating carcinoma ${ }^{27}$. A recent study at MD Anderson Cancer Centre by Chagper et al reported that $18 \%$ of initially node negative by $H \& E$, sentinel nodes were converted to positive by additional examination of SS and performing $\mathrm{IHC}^{28}$. This is supported by other studies in which addition of IHC using cytokeratin antibodies AEI/3 and Pan CK increased the sensitivity of SLNB and converted about $10 \%$ of otherwise lymph node negative patient to lymph node positive ${ }^{29}$. Giuliano reported that $42.3 \%$ of SLN negative by H\&E were positive by use of anticytokeratin antibodies, similar rates were reported by Reintgen and Krag et $\mathrm{al}^{30}$.

\section{Micrometastasis:}

Lymph node metastasis in patient with breast carcinoma represent a broad spectrum of pathology findings ranging from gross disease, to $\mathrm{H} \& \mathrm{E}$ detected macrometastasis $>2 \mathrm{~mm}$, to H\&E and/or IHC detected micrometastasis $<0.2 \mathrm{~mm}$, to single tumour cells found only by IHC. These gradations certainly reflect spectrum of prognostic significant ${ }^{31}$. At one extreme, in 2000, Rosser $^{32}$ and Carter et $\mathrm{al}^{33}$ suggested that the micrometastasis are not biologic metastasis at all but rather the benign transport of breast epithelium by preoperative manipulation of breast and are devoid of clinical significance.

Veronesi et $\mathrm{al}^{34}$ data showed that the sentinel lymph node involved by microfoci $(<0.2 \mathrm{~mm})$ of cancer cells are associated with a considerable rate of metastatic involvement in the remaining axillary lymph nodes. They believe that the presence of microfoci in the SLN is an indication to perform a total ALND. The Memorial Sloan-Kettering Cancer Center has developed a nomogram to provide an accurate risk estimate using pathological characteristics of primary tumour such as tumour size, grade, presence of lymphovascular invasion, multifocality and estrogen receptor status together with SLN characteristics including size of metastasis, number of sentinel node positive and method of detection which may help in predicting non-sentinel node status after SLNB ${ }^{35}$. This nomogram has been validated by study from MD Anderson Cancer Center ${ }^{36}$.

\section{Factors affecting SLN Status:}

SLNB has false negative rate of $0 \%$ to $1.4 \%$ reported in many studies. As a result many medical centres are now using SLNB without a completion ALND in patients who have negative SLN. Bass et $\mathrm{a}^{37}$ described the lymphatic mapping experience at the H.Lee Moffitt Cancer centre and Research Institute from 1994-1999 in 1,147 breast cancer patients and reported $0.83 \%$ false negative rate of SLN. The risk of false negative results is low and may be further reduced if multicentric and multifocal tumours are excluded. Memorial SloanKettering Cancer Center in $2000^{38}$ identified histological features of SLN positive cancers that allowed for the prediction of non-SLN metastasis at shown in Table-3. According to their study the most accurate prediction of non SLN metastasis arose from the combination of three variables significant in univariate analysis: tumour size, lymphovascular invasion, and size of SLN metastasis. The proportion of patients who have metastasis limited to the SLN can be predicted when there is a combination of tumour size $<1.0 \mathrm{~cm}$, the absence of lymphovascular invasion (LVI), and micrometastatic disease $(<0.2 \mathrm{~cm})$ in SLN. It has been shown by other studies too that the tumour size is directly related to the probability of axillary lymph node involvement ${ }^{39}$. For patients with large breast cancer, the role of SLNB is controversial. Early studies of SLNB in large breast cancer patients demonstrated a high (8$18 \%$ ) false negative rate, with the accuracy worsening with the increasing size. Hill AD et al reported that the accuracy of SLNB appeared to diminish with the increasing size ${ }^{40}$. The accuracy was $100 \%$ with T1a,b and decreased to $82 \%$ in T2,T3. This inaccuracy might be caused by alternate lymphatic drainage pathways, or by the increased prevalence of axillary metastatis in patient with large tumour. The tumour size and the size of SLN metastasis alone, as reported by other investigators may not be the only predictors of further residual disease in the axilla. The amount of disease harbored by all the SLN as a whole, reflected by the number of positive SLN and the ratio of positive SLN to total SLN(s), should also be considered as an 
indication of the amount of further disease that may be present in the rest of axillary basin. The presence of more than one positive SLN and /or a ratio of positive SLN(s) to total SLN(s) greater than 0.5 should alert the clinician to a significant possibility of further disease in axillary basin in patients with a positive SLN ${ }^{41}$.

Role of SLNB in patients undergoing Neoadjuvant chemotherapy:

The increasing use of neoadjuvant chemotherapy (CT) in patients with breast cancer has added to the challenge of axillary staging in these patients. It has been reported that SLNB performed prior to neoadjuvant CT eliminates any chemotherapeutic effects on the technical aspects of lymphatic mapping such as sclerosing of lymphatics in response to chemotherapy in the lymph nodes. Obtaining accurate axillary basin information prior to initiation of neoadjuvant chemotherapy may have therapeutic as well as prognostic implications. Jones et al recommended SLNB before neoadjuvant CT in clinically node negative patients at presentation ${ }^{42}$. Their sentinel node identification rates were significantly better when mapping is performed before neoadjuvant CT compared to after completion of CT (100\% Vs $80.6 \%$ ); failure to map correlated with clinically positive nodal disease at presentation and residual disease at ALND. Among patients who mapped successfully after CT, the false negative rate was found to be high (11\%).

The role of SLNB in prophylactic mastectomy (PM): Indications for prophylactic mastectomy range from lobular carcinoma-in situ (LCIS) to a genetic test showing BRCA 1 or 2 mutation, cosmesis and cancer phobia. Occult cancer has been found in upto $5 \%$ of PM cases. Dupont EL et al consequently considered the potential expansion of the use of SLN biopsy in these cases and reported that the non-specific technique of peri-aerolar injection of mapping agents appears to be accurate and sensitive for the identification of sentinel lymph node of patients under going $\mathrm{PM}^{43}$. The lymph node mapping may eliminate the need for axillary dissection if the local disease is detected in the breast following prophylactic mastectomy.
Role of SLNB in clinically positive axillary nodes: By current guidelines, clinically positive axillary nodes are a contraindication of SLNB; but clinical examination is falsely positive in a considerable proportion of patients with either moderately or highly suspicious findings and is by itself insufficient justification for axillary lymph node dissection. Its overall accuracy in various studies varies from 63-68\%.

Follow up and survival analysis:

The follow up of patients after SLNB as for breast cancer patients in general, is for life. The rate of isolated axillary relapse after a negative SLNB is comparable to that after a conventional ALND 1\% or less ${ }^{44}$. The long-term sequel of SLNB remains to be defined, especially the incidence of axillary recurrence. Previous studies also showed quiet infrequent( $0 \%$ to $2.1 \%$ at follow up of $40-180$ months) local recurrence after ALND. Given the low rate of local recurrence after ALND; many studies reported comparably good results in patients with a negative SLNB and no ALND with axillary local recurrence ranging from $0 \%$ to $1.4 \%$ at 14 to 46 months of follow up ${ }^{45}$.

Although SLNB has recently been incorporated into TNM classification, the optimal histopathological work up of SLNs is currently not standardized. Validation trial done in 2005 on survival analysis following SLNB demonstrated its accuracy in staging early breast cancer. It was reported that patients with a positive SLNB result had a significant lower, five year disease free survival rate than patients with a negative SLNB result. This correlated well with established stage II \& III survival information. In conclusion, SLNB is a successful method of staging breast cancer and should be part of the standard of care in this disease.

In summary, SLNB has become the standard of care in the surgical management of a large subset of breast cancer patients. With increasing experience of surgeons and by developing standard protocols by pathologists for handling and processing of these lymph nodes the false negative rates have gone down, sensitivity of identifying micrometastasis has improved greatly, which has made this procedure in the treatment of choice.

Table-I

\begin{tabular}{lccc} 
& \multicolumn{2}{c}{ Sentinel Lymph Node Biopsy } & Using Different Technqiques Weiser et al $^{3}$ \\
Method & SLN found (\%) & Sensitivity & Accuracy \\
\hline Isotope & 92 & 93 & 97 \\
Blue dye & 78 & 91 & 97 \\
Isotope+blue dye & 89 & 97 & 99 \\
\hline
\end{tabular}


Table-II

\begin{tabular}{lcc}
\multicolumn{2}{c}{ Relation of Sectioning Interval with Missed SLN Micrometastasis } \\
Sectioning interval(mm) & $\begin{array}{c}\text { Missed micro- } \\
\text { metastasis(\%) }\end{array}$ & $\begin{array}{c}\text { No. with non sentinel } \\
\text { lymph node metastasis }\end{array}$ \\
\hline 110 & 5.3 & 1 \\
170 & 8.8 & 0 \\
230 & 12.4 & 2 \\
290 & 17.7 & 3 \\
\hline
\end{tabular}

Table-III

Correlation of Primary Tumor Size, Lympho-vascular Invasion (LVI) and SLN Meastasis Size with Non SLN Metastasis ${ }^{3}$

Number of Predictive Factors Present

Tumor Size: $<1.0 \mathrm{~cm}$, LVI negative, SLN metastases $<0.2 \mathrm{~cm}$
Sentinel Lymph Node Only Positive

(\% cases)

\begin{tabular}{rrr}
\hline 3 & 100 \\
2 & 74 \\
1 & 66 \\
0 & $43 \quad 2$ \\
\hline
\end{tabular}

\section{References:}

1. Pisani P, Parkin DM, Bray F, Ferlay JE. Estimates of the worldwide mortality from 25 breast cancer in 1990. Int J Cancer; 83: 870-873.

2. Kim T, Giuliano AE, Lyman GH. Lymphatic mapping and sentinel lymph node biopsy in early-stage breast carcinoma: a metaanalysis. Cancer 2006; 106(1): 416.

3. Fisher B, Wolmark N, Bauer M, Redmond C, Gebhardt M. The accuracy of clinical nodal staging and of limited axillary dissection as a determinant of histologic nodal status in carcinoma of the breast. Surg Gynaecol Obstet 1981; 152(6): 765-72,.

4. Cady B. Is axillary lymph node dissection necessary in routine management of breast cancer? Am J Surg 1997; 3: 246-260.

5. Weiser MR, Montgomery LL, Tan LK, Susnick B, Leung DY, Borgen PL, et al. Lymphphovascular invasion enhances the prediction of non-sentinel node metastasis in breast cancer patients with positive sentinel nodes. Ann Surg Oncol 2001; 8(2): 145-9.

6. Carlo JT, Grant MD, Knox SM, Jones RC, Hamilton CS, Livingstone SA, et al. Survival analysis following sentinel lymph node biopsy: a validation trial demonstrating its accuracy in staging early breast cancer. Proc (Bayl univ Med Cent) 2005; 18(2): 103-107.

7. Giuliano AE, Kirgan DM, Guenther JM, Morton DL. Lymphatic mapping and sentinel lymphadenopathy for breast cancer. Am J Surg 1994; 220: 391-401.

8. Moore KH, Thaler HT, Tan LK, Borgen PI, Cody HS. Immunohistochemically detected tumour cells in the sentinel lymph nodes of patients with breast carcinoma: biologic metastasis or procedural artifact? Cancer 2004; 100(5): 929-34.
9. Cabanas RM. An approach for the treatment of penile carcinoma. Cancer 1977; 39(2): 456-66.

10. Morton DL, Wen DR, Wong JH; Economou JS, Cagle LA, Strom FK, et al. Technical details of intraoperative lymphatic mapping for early stage of melanoma. Arch Surg 1992; 127(4): 392-9.

11. Van der veen H, Hoekstra OS, Paul MA, Cuesta MA, Meijer S. Gamma-Probe- guided sentinel node biopsy to select patient with melanoma for lymphadenopathy. Br J Surg 1994; 8(12): 1769-70.

12. Rubio IT, Korourlan S, Cowan C, et al. Use of touch preparation for intraoperative diagnosis of sentinel lymph node metastasis in breast cancer. Am J Surg 1998; 176: 532-536.

13. O'Hea BJ, Hill AD, El-Shirbiny AM, Yeh SD, Rosen PP, Coit DG, et al. Sentinel lymph node biopsy in breast cancer: initial experience at Memorial Sloan-Kettering cancer centre. J Am Coll Surg 1998; 186(4): 423-7.

14. Cody HS, 3rd. Clinical aspects of sentinel node biopsy. Breast Cancer Res 2001; 3(2): 104-8.

15. Singh Ranger G, Mokbel K. The evolving role of sentinel lymph node biopsy for breast cancer. Eur J Surg oncol 2003; 29(5): 423-5.

16. Linehan DC, Hill AD, Akhurst T, Yeung H, Yeh SD, Tran $\mathrm{KN}$, et al. Intradermal radiocolloid and intra-parenchymal blue dye injection optimize sentinel node identification in breast cancer patients. Ann Surg Oncol 1999; 6(5): 450-4.

17. Knox SM, Ley CA. Comparison of intraparenchymal and intradermal injection for identification of the sentinel node in patients with breast cancer. Proc (Bayl Univ Med Cent) 2002; 15(4): 366-8.

18. Krag D, Weaver D, Ashikaga T, Moffat F, Klimberg VS, Shriver $\mathrm{C}$, et al. The sentinel node in breast cancer- a multicenter validation study N Engl J Med 1998; 339(14): 941-6. 
19. Zurrida S, Galimberti V, Orvieto E, Robertson C, Ballardini $\mathrm{B}$, Cremonesi $\mathrm{M}$, et al. Radioguided sentinel node biopsy to avoid axillary dissection in breast cancer. An Surg oncol 2000; 7(1): 28-31.

20. Brogi E, Torres-Matundan E, Tan LK, Cody HS, 3rd. The results of frozen section, touch preparation and cytological smear are comparable for intraoperative examination of sentinel lymph nodes: a study in 133 breast cancer patients. Ann Surg Oncol 2005; 12(2): 173-80.

21. Krogerus LA, Leidenius MH, Toivonen TS, von Smitten KJ. Towards reasonable workload in diagnosis of sentinel lymph node: comparison of two frozen section methods. Histopathology 2004; 44(1): 29-34.

22. Veronesi U, Paganelli G, Viale G, Galimberti V, Luini A, Zurrida S, et al. Sentinel lymph node biopsy and axillary dissection in breast cancer results in a large series. J Natl Cancer Inst 1999; 91(4): 368-73.

23. Henry-Tillman RS, Korourian S, Rubio AT, Johson A, Mancino N, et al. Intraoperative touch preparation for sentinel lymph node biopsy: a four year experience. Ann Surg Oncol 2002; 94: 333-339.

24. Braun S, Cevatli BS, Assemi C, Janni W, Kentenich CR, Schindlbech C, et al. Comparative analysis of micrometastasis to the bone marrow and lymph nodes of node negative breast cancer patients receiving no adjuvant therapy. J Clin Oncol 2001; 195: 1468-1475.

25. Rao RS, Taylor J, Palmer J, Jennings WC. Breast cancer pseudometastasis in a sentinel lymph node with cytokeratin positive debris. Breast J 2005; 11: 134-137.

26. Viale G, Maiorano E, Mazzarol G, Zurrida S, Galimberti V, Luini A, et al. Histologic detection and clinical implications of micrometastases in axillary sentinel lymph nodes for patients with breast carcinoma. Cancer 2001; 92(6): 1378-84.

27. Intra M, veronesi P, Mazzarol G, Galimberti V, Luini A, Sacchini V, et al. Axillary sentinel lymph node biopsy in patients with pure ductal carcinoma in situ of the breast. Arch Surg 2003; 138(3): 309-13.

28. Chagpar AB, Scoggins CR, Sahwo S, Martin RC, Carlson DJ, Laidley AL, et al. Biopsy type does not influence sentinel lymph node status. Am J Surg 2005; 190((4): 551-6.

29. Czerniecki BJ, Scheff AM, Callans LS, Spitz FR, Bedrosian I, Conant EF, et al. Immunohistochemistry with pancytokeratins improves the sensetivity of sentinel lymph node biopsy in a patient with breast carcinoma. Cancer 1999; 85(5): 1098-103.

30. Reintgen VC Krag D, dewidt-Evert LM, Ruers TJ, Beex LV. Micrometastases in axillary lymph nodes: an increasing classification and treatment dilemma in breast cancer due to the introduction of sentinel lymph node procedure. Breast Cancer Res Treat 2001; 70: 81-88.

31. Specht MC, Fey JV, Borgen Pl, Cody HS, 3rd. Is the clinically positive axilla in breast cancer really a contraindication to sentinel lymph node biopsy? J Am Coll Surg 2005; 200(1): 10-4.

32. Rosser RJ. A point of view: Trauma is the cause of occult micrometastatic breast cancer in sentinel axillary lymph nodes. Breast J 2000; 6(3): 209-212.
33. Carter BA, Jensen RA, Simpson JF, Page DL. Benign transport of breast epithelium into axillary lymph nodes after biopsy. Am J Clin Pathol 2000; 113(2): 259-65.

34. Veronesi U, Paganelli G, Galimberti V, Viale G, Zurrida S, Bedoni M, et al. Sentinel node biopsy to avoid axillary dissection in breast cancer with clinically negative lymph nodes. Lancet 1997; 349: 1864-7.

35. Lambert LA, Ayers GD, Hwang RF, Hunt KK, Ross MI, Kuerer $\mathrm{HM}$, et al. Validation of a breast cancer nomogram for predicting nonsentinel lymph node metastases after a positive sentinel lymph node biopsy. Ann Surg Oncol 2006; 13(3): 310-20.

36. Ollila DW, Neuman HB, Sartor C, Carey LA, Klauber-Demore N. Lymphatic mapping and sentinel lymphadenectomy prior to neoadjuvant chemotherapy in patients with large breast cancers. Am J Surg 2005; 190(3): 371-5.

37. Bass SS, Lyman GH, McCann CR, Ku NN, Berman C, Durand $\mathrm{K}$, et al. Lymphatic mapping and sentinel lymph node biopsy. Breast J 1999; 5(5): 288- 295.

38. Naik AM, Fey J, Gemignani M, Heerdt A, Montogomery L, Petrek J, et al. The risk of axillary relapse after sentinel lymph node biopsy for breast cancer is comparable with that of axillary lymph node dissection: a follow up study of 4008 procedures. Ann Surg 2004; 240(3): 462-8.

39. Klauber-Demore N, Tan LK, Liberman L, Kaptain S, Fey J, Borgen P, et al. Sentinel lymph node biopsy: is it indicated in patients with high risk ductal carcinoma in situ and ductal carcinoma in situ with microinvasion? Ann Surg Oncol 2000; 7(9): 636-42.

40. Hill AD, O’ Hea BJ, EL-Shirbiny AM, Yeh SDJ, Coit DG, et al. Sentinel lymph node biopsy in breast cancer: initial experience at Memorial Sloan- Ketering Cancer. J Am Coll Surg 1998; 186: 423-7.

41. Klevesath MB, Bobrow LG, Pinder SE, Purushotham AD. The value of immunohistochemistry in sentinel lymph node histopathology in breast cancer. Br J Cancer 2005; 92(12): 2201-5.

42. Jones JL, Zabicki K, Christian RL, Gadd MA, Hughes KS, Lesnikoshi BA, et al. A comparison of sentinel node biopsy before and after neoadjuvent chemotherapy: timing is important. Am J Surg 2005; 190(4): 517-20.

43. Dupont EL, Kuhn MA, McCann C, Salud C, Spanton JL, Cox CE. The role of sentinel lymph node biopsy in women undergoing prophylactic mastectomy. Am J Surg 2000; 180(41): 274-77.

44. Dale PS, Williams JTIV. Axillary staging using selective sentinel lymph- adenectomy for patients with invasive breast carcinoma. Ann Surg 1998; 64: 28-31.

45. Giuliano AE, Haigh PI, Brennan M, Hansen NM, Kelley MC, Ye W, Glass EC, Turner RR. Prospective observational study for sentinel lymphadenectomy without further axillary dissection in patients with sentinel node-negative breast cancer. J Clin Oncol 2000; 18: 2553-2559. 